\title{
Effects of MTG and GSH on Human Sperm Motility and DNA Integrity during Vitrification in the Presence of Trehalose
}

\author{
Xiaowei Shi*, Huanhuan Hu*, Guojie Ji, Rui Liu, Jing Zhang, Han Zhang, Pengbo Wang, \\ Bingxin Qin, Bingjie Li, Mingwen Li ${ }^{\#}$
}

Key Laboratory of Fertility Preservation, School of Life Sciences and Technologies, Sanquan College of Xinxiang Medical University, Xinxiang, China

Email: " mingwenli369@163.com

How to cite this paper: Shi, X.W., Hu, H.H., Ji, G.J., Liu, R., Zhang, J., Zhang, H., Wang, P.B., Qin, B.X., Li, B.J. and Li, M.W. (2020) Effects of MTG and GSH on Human Sperm Motility and DNA Integrity during Vitrification in the Presence of Trehalose. Advances in Reproductive Sciences, 8, 71-81. https://doi.org/10.4236/arsci.2020.81007

Received: January 20, 2020

Accepted: February 11, 2020

Published: February 14, 2020

Copyright $\odot 2020$ by author(s) and Scientific Research Publishing Inc. This work is licensed under the Creative Commons Attribution International License (CC BY 4.0).

http://creativecommons.org/licenses/by/4.0/

(c) $\underset{\mathrm{By}}{\text { (i) Open Access }}$

\begin{abstract}
Limited information exists about the effects of antioxidants on sperm motility and DNA integrity during vitrification in humans. This study compared the effects of reduced glutathione (GSH) and monothioglycerol (MTG) at different concentrations on post-thaw sperm motility and DNA integrity after vitrification in humans using $0.25 \mathrm{M}$ trehalose as a cryoprotective agent, and found that supplementation of MTG or GSH at $0.5 \mathrm{mM}$ resulted in significantly higher $(\mathrm{P}<0.05)$ recovery rates of post-thaw total and progressive motilities. GSH was more powerful than MTG at the same concentration in cryoprotecting sperm motility $(38.9 \% \pm 3.6 \%$ vs $32.8 \% \pm 2.4 \%$ compared to the control $26.8 \% \pm 2.1 \%$ in recovery rate of progressive motility), but both had no significant influence on sperm DNA integrity during vitrification. It was concluded that sperm motility is more sensitive to oxidative stress during vitrification than sperm nuclear DNA, and supplementation of MTG or GSH in vitrification medium is beneficial in cryoprotecting sperm motility.
\end{abstract}

\section{Keywords}

Human, Sperm, Vitrification, Trehalose, Antioxidant

\section{Introduction}

Sperm cryopreservation has long been an important strategy in fertility preservation and assisted reproduction. Despite the extensive progress that has been made in the field, decreases in sperm motility and DNA integrity are commonly

${ }^{\star}$ These authors contributed equally to this study. 
observed after cryopreservation [1] [2]. The mechanisms of cryodamage to human sperm have been linked to cold shock, osmotic stress, intracellular ice formation and oxidative stress [1] [3] [4] [5].

Antioxidant supplementation to cryopreservation media has been explored to minimize sperm cryodamage. Studies have shown that antioxidants quercetin, Vitamin E, melatonin, reduced glutathione (GSH) and MitoTEMPO are beneficial to improve post-thaw sperm motility after slow freezing [5] [6] [7] [8] and rapid freezing [9] [10] [11] [12]. Quercetin and GSH were also reported to be efficient in protecting the integrity of sperm nuclear DNA during slow freezing [6] [7] [8] [10] and rapid freezing [9] [11]. However, limited information exists about the use of antioxidants for human sperm cryopreservation by vitrification, although vitrification using sucrose or trehalose as non-permeating cryoprotective agents (CPA) has been demonstrated to be superior to slow freezing in cryopreserving sperm motility and DNA integrity [13] [14] [15]. So far, only butylhydroxytoluene (BHT, a synthetic analogue of vitamin E) and hypotaurine were reported to be beneficial in vitrifying human sperm motility and DNA integrity [16] [17]. In addition, antioxidant monothioglycerol (MTG) has been found to be extremely effective in cryoprotecting mouse sperm motility, DNA integrity and fertilization ability [18] [19] [20]; however, this antioxidant has not yet been tested for human sperm cryopreservation.

Thus, the objective of the present study was to examine and compare the efficacies of the antioxidants MTG and GSH in cryoprotecting sperm motility and nuclear DNA integrity during vitrification in humans using trehalose as a CPA.

\section{Materials and Methods}

\subsection{Materials}

All chemicals and reagents were purchased from Sigma-Aldrich Co. (Shanghai, China) unless otherwise stated. Human tubal fluid (HTF) medium containing 5 $\mathrm{mg} / \mathrm{ml}$ human serum albumin (HSA) was prepared according to the method of Quinn et al. [21] GSH and MTG stock solutions were prepared in HTF medium.

\subsection{Experimental Designs}

Three experiments were performed, and each experiment was repeated at least 3 times using normozoospermic samples from different donors. Experiments 1 and 2 were to study the cryoprotective effects of MTG and GSH, respectively, during vitrification on recovery rates of sperm total motility and progressive sperm motility and sperm nuclear DNA integrity. Experiment 3 was to compare the cryoprotective effects of MTG and GSH on sperm motility and DNA integrity during vitrification.

\subsection{Semen Preparation}

Semen samples were obtained by masturbation in a private room near the laboratory from 15 healthy volunteer donors from the ages of 21 to 35 years old after 
3 - 7 days of sexual abstinence. Written informed consent was obtained from all semen donors before the procedure. The study was approved by the Ethics Committee of Xinxiang Medical University. The semen collection, liquefaction and analysis for volume, sperm concentration, motility and morphology were carried out according to the guidelines and protocols recommended by the World Health Organization (WHO, 2010) [22]. Ejaculates with volume $<2 \mathrm{ml}$, concentration $<5 \times 10^{7} / \mathrm{ml}$, progressive motility $<50 \%$ and normal sperm morphology $<$ $30 \%$ were excluded from the study. Upon liquification at $37^{\circ} \mathrm{C}$, semen was diluted with $5 \% \mathrm{CO}_{2}$ pre-equilibrated warm $\mathrm{HTF}$ medium at 1:2 ratio and then the sperm were washed twice by centrifugations ( $400 \times \mathrm{g}$ for $10 \mathrm{~min}$ each) and resuspensions. Washed sperm were incubated at $37^{\circ} \mathrm{C}$ in $5 \% \mathrm{CO}_{2}$ prior to cryopreservation.

\subsection{Sperm Cryopreservation}

Washed sperm in HTF medium from each ejaculate were assessed immediately for sperm quality, and then mixed thoroughly and divided into equal aliquots using a large orifice pipet tip. Each aliquot was diluted 1:1 with aqueous solution of trehalose at $0.5 \mathrm{~mol} / \mathrm{L}$, so that the final concentration of sperm was $15-20 \times$ $10^{6} / \mathrm{ml}$ and the final concentration of trehalose was $0.25 \mathrm{~mol} / \mathrm{L}$.

Immediately, each of the sperm aliquots was supplemented with different concentrations of an antioxidant (MTG or GSH) by adding $2.5 \mu \mathrm{L}$ of $200 \times$ stock solution of each concentration to be tested to each sperm aliquot and mixing thoroughly. The concentrations tested for each of the two antioxidants were selected on the basis of previous studies [6] [8] [19] [20]. Then, the sperm samples were loaded into straw-in-straws systems (Figure 1). The straw-in-straw system was made by loading a shortened ( $2 \mathrm{~cm}$ cut off from the open end) standard 0.25 mL straw (IMV Technologies, USA, catalog \# 005565) with $50 \mu \mathrm{l}$ sperm sample by aspiration to the middle part of the straw. Then, the loaded straw was placed inside a standard 0.5mL straw (IMV Technologies, USA, catalog \# 005569) with their plug ends on the same side. After the plug end of the $0.5 \mathrm{~mL}$ straw was heat-sealed, the open ends of both the 0.25 and $0.5 \mathrm{~mL}$ straws were heat-sealed together using an impulse sealer. Finally, the sperm samples were equilibrated in vitrification media for $10 \mathrm{~min}$ at room temperature before vitrification.

Vitrification was performed by submerging sperm samples directly and horizontally into liquid nitrogen $\left(\mathrm{LN} 2,-196^{\circ} \mathrm{C}\right)$. Sperm samples were stored in LN2 for 1 week before analysis. All cryopreserved sperm samples were thawed by submerging a straw-in-straw system in a $37^{\circ} \mathrm{C}$ water bath for $2 \mathrm{~min}$ before the heat seals at both ends were cut off and the sperm suspension was expelled into 1 mL pre-warmed HEPES-buffered HTF medium for dilution.

Seal

Seal

\section{Plug}

Plug

$50 \mu \mathrm{L}$

Figure 1. The straw-in-straw system loaded with $50 \mu \mathrm{l}$ sperm and heat-sealed at both ends. The straw-in-straw system was made by inserting a $0.25 \mathrm{~mL}$ straw loaded with sperm into a $0.5 \mathrm{~mL}$ straw with their plug ends placed on the same side. 


\subsection{Sperm Concentration and Motility Assessments}

Sperm concentration, total motility (\% of motile sperm) and progressive motility (\% of sperm with curvilinear velocity $>25 \mu \mathrm{m} / \mathrm{s}$ and straightness $\geq 0.8$ ) at $37^{\circ} \mathrm{C}$ were measured immediately before vitrification and post-thaw using counting chambers with $20-\mu \mathrm{m}$ depth and a WLJY-9000 computer-assisted sperm analyzer (Weili New Century Science \& Tech, Beijing, China). At least 2000 sperm per sample from randomly selected fields were examined. Sperm motility recovery rates including total motility recovery rate and progressive motility recovery rate were calculated and used to evaluate the cryoprotective effects of different treatments during vitrification. Motility recovery rate $=$ (post-thaw motility $\div$ pre-freeze motility) $\times 100 \%$.

\subsection{Sperm DNA Damage Assessments}

The nuclear DNA integrity of post-thawed sperm samples was assessed by the sperm chromatin dispersion (SCD) test as described by Fernández et al. [23] with some modifications. Briefly, $30 \mu \mathrm{L}$ of sperm suspension from a sample was mixed with $70 \mu \mathrm{L}$ of $1 \%$ low melting agarose in $\mathrm{Ca} / \mathrm{Mg}$-free $\mathrm{PBS}$ at $37^{\circ} \mathrm{C}$, and then $30 \mu \mathrm{l}$ of mixture was added onto a slide and spread with a $22 \times 22 \mathrm{~mm}$ cover glass. After solidification of the agarose at $4^{\circ} \mathrm{C}$ for $5 \mathrm{~min}$, the cover glass was removed, and the slide was immersed in $0.08 \mathrm{~mol} / \mathrm{L} \mathrm{HCl}$ for $7 \mathrm{~min}$ in the dark at room temperature. Then, the slide was treated in neutralizing and lysing solution containing $0.4 \mathrm{~mol} / \mathrm{L}$ Tris- $\mathrm{HCl}, \mathrm{pH} 7.5,0.1 \mathrm{~mol} / \mathrm{L}$ dithiothreitol (DTT), $0.5 \%$ sodium dodecyl sulfate (SDS) and $0.005 \mathrm{~mol} / \mathrm{L}$ ethylenediaminetetraacetic acid disodium salt solution (EDTA) for $20 \mathrm{~min}$ at room temperature. Further, the slide was dehydrated in 70\%, 90\% and 100\% ethanol and air-dried. After being mounted with VECTASHIELD ${ }^{\circledR}$ containing DAPI (Vector Laboratories, Inc., USA), the slide was scored under an epifluorescence microscope (Nikon Instruments, Japan) at 1000× magnification. At least 200 sperm were examined per sample, and sperm DNA fragmentation index (DFI), i.e. the percentage of sperm with non-dispersed chromatin (with fragmented DNA), was calculated (Figure 2).

\subsection{Statistical Analysis}

GraphPad Prism 5 (GraphPad Software, Inc., San Diego, USA) was used for statistical analysis. Sperm motility recovery rates and percentages of sperm with DNA fragmentation were arcsine transformed, and then group differences were detected by $\mathrm{t}$ tests and one-way ANOVA followed by Tukey HSD tests, where $\mathrm{P}$ $<0.05$ was considered significant. Data are expressed as mean $(\mathrm{M}) \pm$ standard deviation (SD).

\section{Results}

\subsection{Cryoprotective Effect of MTG on Sperm Motility and DNA Integrity}

In this experiment, sperm were exposed to 0 (Ctrl), $0.25,0.5,1.0$ and $2.0 \mathrm{mM}$ 


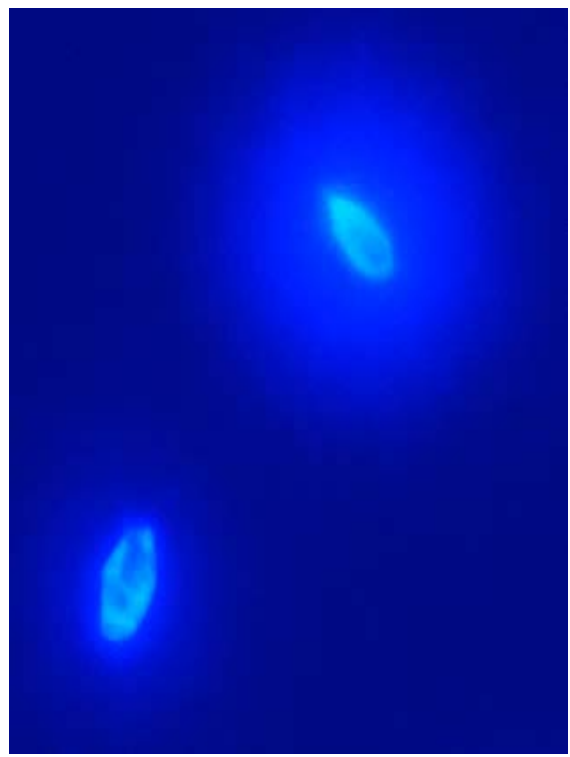

Figure 2. Human sperm SCD test. The bottom sperm with small halo indicating DNA fragmentation and the upper sperm with large halo indicating intact DNA. Original magnification $1000 \times$.

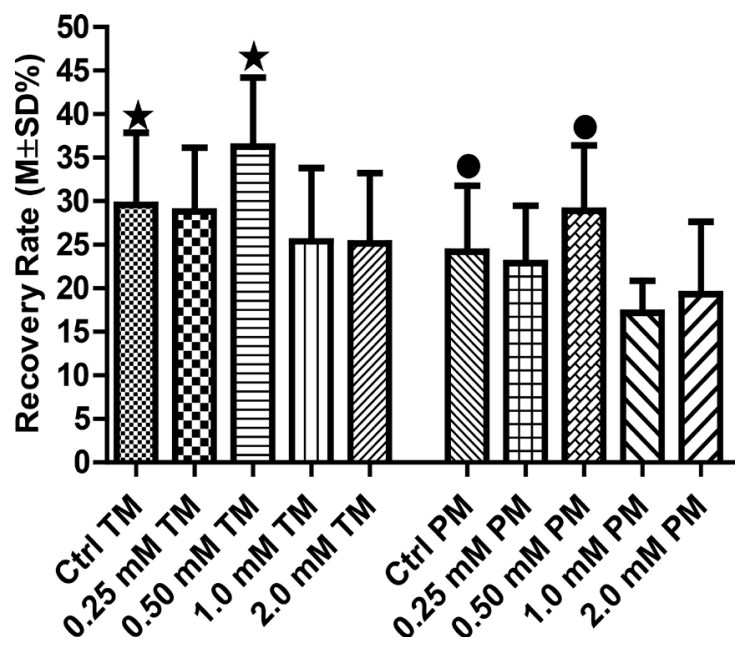

Figure 3. Effect of different concentrations of MTG on the recovery rate of sperm total motility (TM) and progressive motility $(\mathrm{PM}) . \mathrm{P}<0.05$ between bars with the same symbols.

MTG during vitrification. The data shown in Figure 3 indicate that MTG at 0.5 $\mathrm{mM}$ significantly $(\mathrm{P}<0.05)$ protected sperm total motility $(36.3 \% \pm 7.9 \%$ vs $29.6 \% \pm 8.3 \%$ in recovery rate) and progressive motility $(28.9 \% \pm 7.5 \%$ vs $24.2 \%$ $\pm 7.6 \%$ in recovery rate) compared to the controls, but lower and higher concentrations of MTG tested had no significant effect on both total and progressive motilities $(\mathrm{P}>0.05)$.

We also measured DFI of post-thawed sperm and the data summarized in Figure 4 indicates that MTG had no significant effect $(P>0.05)$ on sperm DNA integrity during vitrification at the concentrations tested compared to the control DFI $(13.5 \% \pm 1.8 \%)$. 


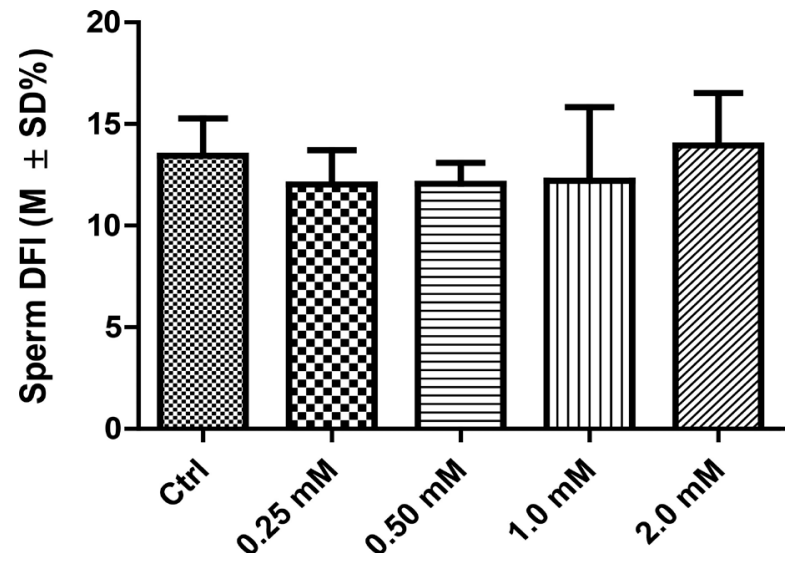

Figure 4. Effect of different concentrations of MTG on post-thaw sperm DFI. No significant difference was found among the tested concentrations.

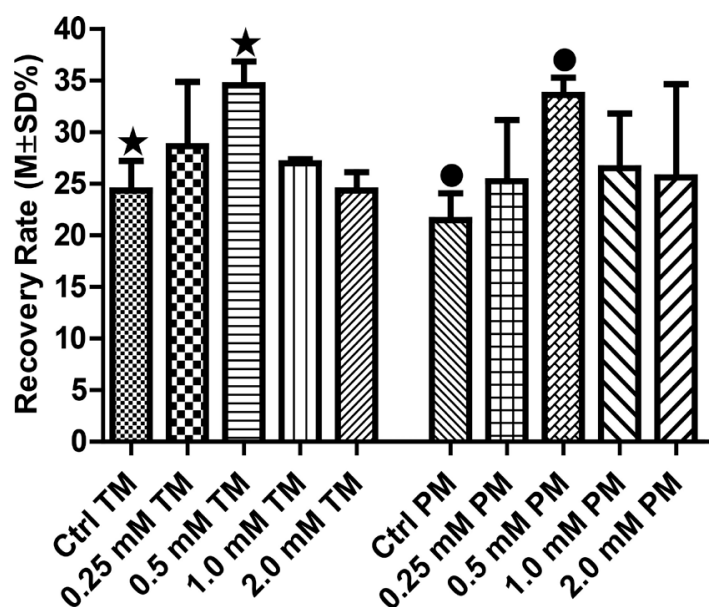

Figure 5. Effect of different concentrations of GSH on the recovery rate of sperm total motility $(\mathrm{TM})$ and progressive motility $(\mathrm{PM}) . \mathrm{P}<0.05$ between the bars with the same symbols.

\subsection{Cryoprotective Effect of GSH on Sperm Motility and DNA Integrity}

In this experiment, sperm were exposed to $0(\mathrm{Ctrl}), 0.25,0.5,1.0$ and $2.0 \mathrm{mM}$ of GSH during vitrification. The data summarized in Figure 5 show that $0.5 \mathrm{mM}$ GSH was significantly efficient $(\mathrm{P}<0.05)$ in cryoprotecting both total motility $(34.5 \% \pm 2.3 \%$ vs $24.4 \% \pm 2.9 \%$ in recovery rate) and progressive motility (recovery rate $33.6 \% \pm 1.7 \%$ vs $21.5 \% \pm 2.6 \%$ ) compared to the controls, but GSH had no significant effect on cryoprotecting sperm nuclear DNA integrity $(\mathrm{P}>0.05$, Figure 6$)$ at the concentrations tested compared to the control DFI $(8.4 \% \pm 2.8 \%)$.

\subsection{Comparison of MTG and GSH in Cryoprotecting Sperm Motility}

To compare the cryoprotecting effects of MTG and GSH on sperm motility, sperm samples were exposed to MTG or GSH at the same concentration (0.5 $\mathrm{mM}$ ) for vitrification. The post-thaw recovery rates of sperm total motility and progressive motility results are summarized in Figure 7. Both MTG and GSH 


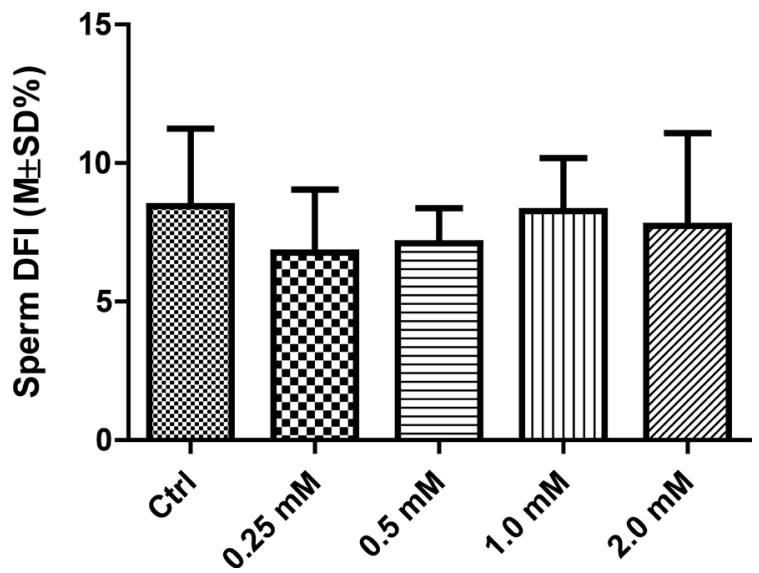

Figure 6. Effect of different concentrations of GSH on post-thaw sperm DFI. No significant difference was found among the tested concentrations.

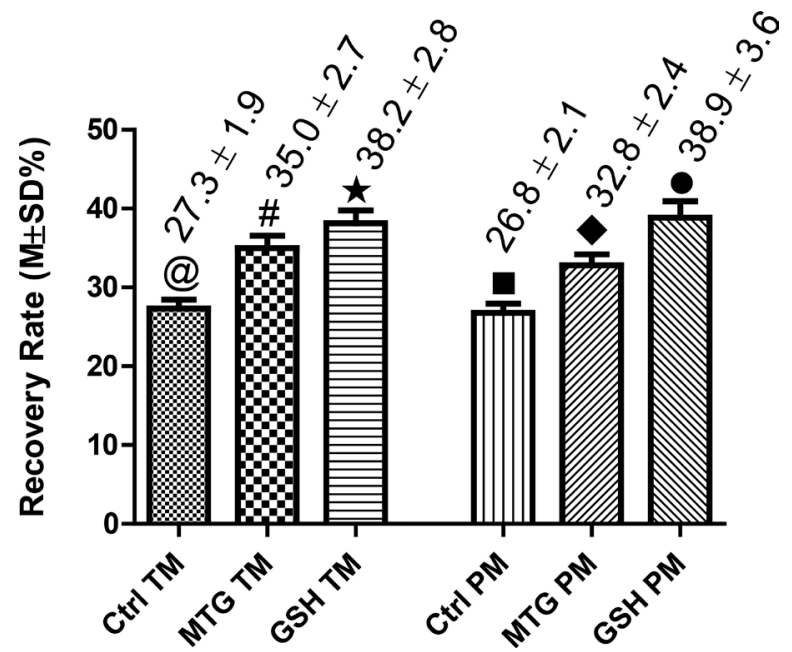

Figure 7. Comparison of MTG $(0.5 \mathrm{mM})$ and GSH $(0.5 \mathrm{mM})$ in cryoprotecting sperm total motility $(\mathrm{TM})$ and progressive motility $(\mathrm{PM}) . \mathrm{P}<0.05$ between bars with different symbols in the same group (TM or PM).

had significant cryoprotective effects $(\mathrm{P}<0.05)$ on total motility and progressive motility compared to the controls, and GSH was more efficient $(\mathrm{P}<0.05)$ than MTG.

\section{Discussion}

Sperm cryopreservation significantly reduces motility, DNA integrity and fertility in humans [1] [2]. The mechanisms of sperm cryodamage are most likely multifactorial, but oxidative stress has been recognized as a significant cause [1]. Excess reactive oxygen species (ROS) produced during cryopreservation impair sperm motility and fertilization ability through membrane lipid peroxidation, protein thiol group oxidation and other mechanisms [24]. Oxidative stress can also induce DNA fragmentation in sperm nuclear genome [4] [16] [17].

Oxidative stress is caused by any imbalance between prooxidants and antioxidants in which the former prevail. The presence of an appropriate concentra- 
tion of antioxidants in sperm freezing medium can neutralize the oxidative stress; however, excessive antioxidants can stop the normal sperm functions, including sperm motility and fertility, associated with ROS [25] [26] [27], and this is most likely why we found in the present study that MTG and GSH at concentrations higher than $0.5 \mathrm{mM}$ had no protective effects on sperm motility during vitrification.

GSH at $1 \mathrm{mM}$ and $5 \mathrm{mM}$ was reported to cryoprotect human sperm motility during slow freezing [6] [7] [28], but in the present study we found $0.5 \mathrm{mM}$ was the optimal concentration for human sperm vitrification. It is possible that a larger amount of ROS is produced during slow freezing than vitrification (ultra-rapid freezing) and a higher concentration of antioxidant is thus needed to protect sperm from ROS attacks.

Antioxidant MTG at $0.477 \mathrm{mM}$ or $0.5 \mathrm{mM}$ has been demonstrated to be effective in protecting mouse sperm motility during cryopreservation by rapid freezing [18] [19] [20]. In the present report, we found for the first time that MTG at $0.5 \mathrm{mM}$ is also effective in protecting human sperm motility during vitrification. We also found MTG at $0.5 \mathrm{mM}$ was less efficient in cryoprotecting both total motility and progressive motility than GSH at the same concentration.

Interestingly, we did not find significant effects of MTG and GSH on cryoprotecting human sperm DNA integrity during vitrification in the present study, and this observation is consistent with the results of Taylor et al. (2009) [29] and Banihani et al. (2014) [30] that vitamin E and L-carnitine did not affect human sperm DNA during slow freezing. Recently, antioxidants GSH, vitamin E and BHT were also found to be inefficient in cryoprotecting sperm DNA integrity during rapid freezing in boars [31]. However, studies of Merino et al. (2015) [16] and Seify et al. (2019) [17] reported that antioxidants BHT and hypotaurine were beneficial in cryoprotecting human sperm DNA during vitrification of human sperm. GSH at high concentrations $(5 \mathrm{mM})$ were also reported to be efficient in cryoprotecting human sperm DNA integrity during slow freezing [6] [7] [8].

\section{Conclusion}

In conclusion, this study compared the effects of GSH and MTG on post-thaw sperm motility and DNA integrity after vitrification in humans and found that supplementation of MTG or GSH at $0.5 \mathrm{mM}$ resulted in significantly higher post thaw total and progressive motilities. GSH is more powerful than MTG at the same concentration in protecting sperm motility, but neither had any significant influence on sperm DNA integrity during vitrification.

\section{Acknowledgements}

This work was supported by the National Natural Science Foundation of China (the Joint Funds of NSFC and Henan Province, Award No. U1604179). 


\section{Authors' Contributions}

All authors have accepted responsibility for the entire content of this submitted manuscript and approved its submission.

\section{Conflicts of Interest}

The authors declare no conflicts of interest regarding the publication of this paper.

\section{References}

[1] Amidi, F., Pazhohan, A., Shabani Nashtaei, M., Khodarahmian, M. and Nekoonam, S. (2016) The Role of Antioxidants in Sperm Freezing: A Review. Cell and Tissue Banking, 17, 745-756. https://doi.org/10.1007/s10561-016-9566-5

[2] Kumar, A., Prasad, J.K., Srivastava, N. and Ghosh, S.K. (2019) Strategies to Minimize Various Stress-Related Freeze-Thaw Damages during Conventional Cryopreservation of Mammalian Spermatozoa. Biopreservation and Biobanking, 17. https://doi.org/10.1089/bio.2019.0037

[3] Chatterjee, S. and Gagnon, C. (2001) Production of Reactive Oxygen Species by Spermatozoa Undergoing Cooling, Freezing, and Thawing. Molecular Reproduction and Development, 59, 451-458. https://doi.org/10.1002/mrd.1052

[4] Thomson, L.K., Fleming, S.D., Aitken, R.J., De Iuliis, G.N., Zieschang, J.A. and Clark, A.M. (2009) Cryopreservation-Induced Human Sperm DNA Damage Is Predominantly Mediated by Oxidative Stress rather than Apoptosis. Human Reproduction, 24, 2061-2070. https://doi.org/10.1093/humrep/dep214

[5] Karimfar, M.H., Niazvand, F., Haghani, K., Ghafourian, S., Shirazi, R. and Bakhtiyari, S. (2015) The Protective Effects of Melatonin against Cryopreservation-Induced Oxidative Stress in Human Sperm. International Journal of Immunopathology and Pharmacology, 28, 69-76. https://doi.org/10.1177/0394632015572080

[6] Varghese, A.C., Das, S., Bhattacharya, A.K., Bhattacharya, S.M., Mandal, M. and Agarwal, A. (2005) Effect of Cryoprotective Additives-Reduced Glutathione, Acetyl-L-Carnitine on Sperm Membrane Lipid Peroxidation, DNA Integrity and Recovery of Motile Human Sperm. Fertility and Sterility, 84, S410-S411. https://doi.org/10.1016/j.fertnstert.2005.07.1073

[7] Kalthur, G., Raj, S., Thiyagarajan, A., Kumar, S., Kumar, P. and Adiga, S.K. (2011) Vitamin E Supplementation in Semen-Freezing Medium Improves the Motility and Protects Sperm from Freeze-Thaw-Induced DNA Damage. Fertility and Sterility, 95, 1149-1151. https://doi.org/10.1016/j.fertnstert.2010.10.005

[8] Ghorbani, M., Vatannejad, A., Khodadadi, I., Amiri, I. and Tavilani, H. (2016) Protective Effects of Glutathione Supplementation against Oxidative Stress during Cryopreservation of Human Spermatozoa. CryoLetters, 37, 34-40.

[9] Zribi, N., Chakroun, N.F., Ben Abdallah, F., Elleuch, H., Sellami, A., Gargouri, J., Rebai, T., Fakhfakh, F. and Keskes, L.A. (2012) Effect of Freezing-Thawing Process and Quercetin on Human Sperm Survival and DNA Integrity. Cryobiology, 65, 326-331. https://doi.org/10.1016/j.cryobiol.2012.09.003

[10] Nekoonam, S., Nashtaei, M.S., Naji, M., Zangi, B.M. and Amidi, F. (2016) Effect of Trolox on Sperm Quality in Normozospermia and Oligozospermia during Cryopreservation. Cryobiology, 72, 106-111. https://doi.org/10.1016/j.cryobiol.2016.02.008 
[11] Azadi, L., Tavalaee, M., Deemeh, M.R., Arbabian, M. and Nasr-Esfahani, M. (2017) Effects of Tempol and Quercetin on Human Sperm Function after Cryopreservation. CryoLetters, 38, 29-36.

[12] Lu, X., Zhang, Y., Bai, H., Liu, J., Li, J. and Wu, B. (2018) Mitochondria-Targeted Antioxidant MitoTEMPO Improves the Post-Thaw Sperm Quality. Cryobiology, 80, 26-29. https://doi.org/10.1016/j.cryobiol.2017.12.009

[13] Schulz, M., Risopatrón, J., Matus, G., Pineda, E., Rojas, C., Isachenko, V., Isachenko, E. and Sánchez, R. (2017) Trehalose Sustains a Higher Post-Thaw Sperm Motility than Sucrose in Vitrified Human Sperm. Andrologia, 49, e12757. https://doi.org/10.1111/and.12757

[14] Isachenko, V., Rahimi, G., Mallmann, P., Sanchez, R. and Isachenko, E. (2017) Technologies of Cryoprotectant-Free Vitrification of Human Spermatozoa: Asepticity as Criterion of Effectiveness. Andrology, 5, 1055-1063.

https://doi.org/10.1111/andr.12414

[15] Li, Y.X., Zhou, L., Lv, M.Q., Ge, P., Liu, Y.C. and Zhou, D.X. (2019) Vitrification and Conventional Freezing Methods in Sperm Cryopreservation: A Systematic Review and Meta-Analysis. The European Journal of Obstetrics \& Gynecology and Reproductive Biology, 233, 84-92. https://doi.org/10.1016/j.ejogrb.2018.11.028

[16] Merino, O., Aguagüiña, W.E., Esponda, P., Risopatrón, J., Isachenko, E., Isachenko, V. and Sánchez, R. (2015) Protective Effect of Butylated Hydroxytoluene on Sperm Function in Human Spermatozoa Cryopreserved by Vitrification Technique. Andrologia, 47, 186-193. https://doi.org/10.1111/and.12246

[17] Seify, M., Zarabadipour, M., Ghaleno, L.R., Alizadeh, A. and Rezazadeh Valojerdi, M. (2019) The Anti-Oxidant Roles of Taurine and Hypotaurine on Acrosome Integrity, HBA and HSPA2 of the Human Sperm during Vitrification and Post Warming in Two Different Temperature. Cryobiology, 90, 89-95.

https://doi.org/10.1016/j.cryobiol.2019.07.004

[18] Ostermeier, G.C., Wiles, M.V., Farley, J.S. and Taft, R.A. (2008) Conserving, Distributing and Managing Genetically Modified Mouse Lines by Sperm Cryopreservation. PLoS ONE, 3, e2792. https://doi.org/10.1371/journal.pone.0002792

[19] Li, M.W., Vallelunga, J.M., Kinchen, K.L., Rink, K.L., Zarrabi, J., Shamamian, A.O. and Lloyd, K.C. (2014) IVF Recovery of Mutant Mouse Lines Using Sperm Cryopreserved with MTG in Cryovials. CryoLetters, 35,145-153.

[20] Shi, X., Hu, H., Ji, G., Zhang, J., Liu, R., Zhang, H. and Li, M. (2018) Protective Effect of Sucrose and Antioxidants on Cryopreservation of Sperm Motility and DNA Integrity in C57BL/6 Mice. Biopreservation and Biobanking, 16. https://doi.org/10.1089/bio.2018.0037

[21] Quinn, P., Kerin, J.F. and Warnes, G.M. (1985) Improved Pregnancy Rate in Human in Vitro Fertilization with the Use of a Medium Based on the Composition of Human Tubal Fluid. Fertility and Sterility, 44, 493-498. https://doi.org/10.1016/S0015-0282(16)48918-1

[22] WHO (2010) WHO Laboratory Manual for the Examination of Human Semen and Semen-Cervical Mucus Interaction. 5th Edition, Cambridge University Press, Cambridge, UK.

[23] Fernández, J.L., Muriel, L., Goyanes, V., Segrelles, E., Gosálvez, J., Enciso, M., LaFromboise, M. and De Jonge, C. (2005) Simple Determination of Human Sperm DNA Fragmentation with an Improved Sperm Chromatin Dispersion Test. Fertility and Sterility, 84, 833-842. https://doi.org/10.1016/j.fertnstert.2004.11.089

[24] O’Flaherty, C. and Matsushita-Fournier, D. (2017) Reactive Oxygen Species and 
Protein Modifications in Spermatozoa. Biology of Reproduction, 97, 577-585. https://doi.org/10.1093/biolre/iox104

[25] Kodama, H., Kuribayashi, Y. and Gagnon, C. (1996) Effect of Sperm Lipid Peroxidation on Fertilization. Journal of Andrology, 17, 151-157.

[26] Griveau, J.F. and Le Lannou, D. (1997) Reactive Oxygen Species and Human Spermatozoa: Physiology and Pathology. International Journal of Andrology, 20, 61-69. https://doi.org/10.1046/j.1365-2605.1997.00044.x

[27] Pons-Rejraji, H., Sion, B., Saez, F., Brugnon, F., Janny, L. and Grizard, G. (2009) Role of Reactive Oxygen Species (ROS) on Human Spermatozoa and Male Infertility. Gynécologie Obstétrique \& Fertilité, 37, 529-535. https://doi.org/10.1016/j.gyobfe.2009.04.015

[28] Gadea, J., Molla, M., Selles, E., Marco, M., Garcia-Vazquez, F.A. and Gardon, J.C. (2011) Reduced Glutathione Content in Human Sperm Is Decreased after Cryopreservation: Effect of the Addition of Reduced Glutathione to the Freezing and Thawing Extenders. Cryobiology, 62, 40-46. https://doi.org/10.1016/j.cryobiol.2010.12.001

[29] Taylor, K., Roberts, P., Sanders, K. and Burton, P. (2009) Effect of Antioxidant Supplementation of Cryopreservation Medium on Post-Thaw Integrity of Human Spermatozoa. Reproductive BioMedicine Online, 18, 184-189. https://doi.org/10.1016/S1472-6483(10)60254-4

[30] Banihani, S., Agarwal, A., Sharma, R. and Bayachou, M. (2014) Cryoprotective Effect of L-Carnitine on Motility, Vitality and DNA Oxidation of Human Spermatozoa. Andrologia, 46, 637-641. https://doi.org/10.1111/and.12130

[31] Baishya, S.K., Biswas, R.K., Govindasamy, K., Deka, B.C., Sinha, S. and Singh, M. (2018) Effect of Reduced Glutathione, Water Soluble Vitamin E Analogue and Butylated Hydroxytoluene on the Post Thaw Characteristics of Boar Spermatozoa. CryoLetters, 39, 227-234. 